УДК 616.24-006:616-079.1

Мурашко Д.И., Таганович А.Д., Ковганко Н.Н.

Белорусский государственный медицинский университет, Минск, Беларусь

Murashka D., Tahanovich A., Kauhanka M.

Belarusian state medical university, Minsk, Belarus

\title{
Диагностическая значимость
} определения уровня хемокина CXCL8 и его рецептора CXCR1 в крови пациентов с немелкоклеточным раком легкого

\author{
Diagnostic Significance of Determination of the Level \\ of the Chemokine CXCL8 and its Receptor CXCR1 \\ in the Blood of Patients with Non-Small Cell Lung Cancer
}

Резюме

Введение. Немелкоклеточный рак легкого (НМРЛ) - одна из ведущих причин смертности от злокачественных новообразований. Заболевание включает в себя два основных гистологических типа: аденокарциному (АК) и плоскоклеточный рак (ПКРЛ). В настоящее время отсутствуют информативные показатели в крови, которые позволили бы судить о наличии и распространенности НМРЛ.

Цель. Изучить количественные взаимоотношения концентрации хемокина CXCL8 и его рецептора CXCR1 в крови пациентов с АК и ПКРЛ с тем, чтобы на этой основе оценить возможность их использования в диагностике НМРЛ.

Материалы и методы. Материалом служила кровь 109 пациентов с ПКРЛ, 94 пациентов с AK, 13 человек с гамартомой легкого и 40 здоровых людей. Концентрацию CXCL8 в сыворотке крови определяли методом ИФА. Относительное количество клеток лейкоцитарного ряда, снабженных рецептором CXCR1, и плотность его расположения в них (MFI) измеряли методом проточной цитометрии.

Результаты. Уровень CXCL8 в сыворотке крови, доля гранулоцитов и лимфоцитов с рецептором CXCR1 и MFI CXCR1 в лейкоцитах пациентов С АК и ПКРЛ значительно превышает таковые у здоровых людей и пациентов с гамартомой. MFI CXCR1 (гранулоциты) и доля лимфоцитов с CXCR1 имеют коррелятивную связь средней силы с АК и ПКРЛ. Доля гранулоцитов и лимфоцитов с рецептором CXCR1 и MFI CXCR1 (гранулоциты) выше у пациентов с метастазами в регионарные лимфоузлы, а концентрация CXCL8, доля лимфоцитов C CXCR1 и MFI CXCR1 (лимфоциты) - с отдаленными метастазами. Получены доказательства диагностической ценности определения MFI CXCR1 (гранулоциты) у пациентов с I-II стадиями НМРЛ (диагностическая чувствительность для рассчитанного порогового значения - 98,7\%, специфичность - 71,1\%) и c III-IV стадиями (чувствительность - 87,5\%, специфичность - 93,3\%), превышающей эффективность определения с этой целью концентрации CYFRA 21-1 в сыворотке крови.

Заключение. В крови пациентов с АК и ПКРЛ отсутствует существенная разница уровня CXCL8 и CXCR1. Изменение MFI CXCR1 (гранулоциты) зависит от стадии НМРЛ, размеров опухоли, 
наличия метастазов и имеет более выраженный характер, чем изменение концентрации CYFRA 21-1 в сыворотке крови. Полученные данные позволяют рекомендовать определение MFI CXCR1 (гранулоциты) крови к использованию в диагностике НМРЛ.

Ключевые слова: хемокин, рецептор, CXCL8, CXCR1, немелкоклеточный рак легкого, аденокарцинома, плоскоклеточный рак, кровь.

\section{Abstract}

Background. Non-small cell lung cancer (NSCLC) is one of the leading causes of death from malignant neoplasms. The disease includes two main histological types: adenocarcinoma (AC) and squamous cell carcinoma (SCC). Currently, there are no informative blood indicators that would allow us to estimate the presence and prevalence of NSCLC.

Purpose. To study the quantitative relationship between blood levels of chemokine CXCL8 and its receptor CXCR1 in AC and SCC patients in order to assess the possibility of its use in NSCLC diagnostics.

Materials and methods. The material was the blood of 109 SCC patients, 94 AC patients, 13 people with lung hamartoma, and 40 healthy people. CXCL8 serum level was determined with ELISA. The relative number of leukocytes equipped with CXCR1 and the density of its location in the cells (MFI) were measured with flow cytometry.

Results. The CXCL8 serum level, proportion of granulocytes and lymphocytes containing CXCR1 and MFI CXCR1 in AC and SCC patient's white blood cells significantly exceeded those in healthy people and patients with lung hamartoma. MFI CXCR1 (granulocytes) and proportion of lymphocytes equipped with CXCR1 have a medium-strength correlation with AC and SCC. The proportion of granulocytes and lymphocytes containing CXCR1, MFI CXCR1 (granulocytes) is higher in patients with lymph node metastases. The concentration of CXCL8, proportion of lymphocytes equipped with CXCR1, MFI CXCR1 (lymphocytes) is higher in patients with distant metastases. There is the evidence of high diagnostic value of determination of MFI CXCR1 (granulocytes) in patients with the stages I-II NSCLC (sensitivity for calculated cutoff value $-98,7 \%$, specificity $-71,1 \%$ ) and the stages III-IV (sensitivity - 87,5\%, specificity - 93,3\%), significantly exceeding the effectiveness of determination of CYFRA 21-1 serum level for this purpose.

Conclusion. There is no significant difference in CXCL8 and CXCR1 levels in patients with AC and SCC. MFI CXCR1 (granulocytes) depends on staging of NSCLC, tumor size, regional and distant metastases; its change is more manifested than CYFRA 21-1 serum level. The obtained data allow us to recommend determination of MFI CXCR1 (granulocytes) for the use in the NSCLC diagnostics. Keywords: chemokine, receptor, CXCL8, CXCR1, non-small cell lung cancer, adenocarcinoma, squamous cell carcinoma, blood.

\section{- ВВЕДЕНИЕ}

Рак легкого является самым распространенным злокачественным новообразованием в мире. В 2018 г. зарегистрировано 2,1 млн новых случаев рака легкого (12\% от всех диагностируемых случаев рака) и 1,8 млн смертей от этого заболевания, что составляет пятую часть от смертности вследствие онкологических заболеваний [1]. Более половины пациентов с раком легкого погибают в течение года с момента постановки диагноза [2].

В структуре заболеваемости раком легкого 85\% принадлежит немелкоклеточному раку (НМРЛ), который подразделяется на аденокарциному (АК, 40\% случаев рака легкого), плоскоклеточный рак легкого 
(ПКРЛ, 30\% случаев) и крупноклеточную карциному (10\%) [2]. НМРЛ характеризуется практически бессимптомным течением, что затрудняет своевременную диагностику заболевания. Лишь у 25\% всех пациентов диагноз устанавливается на ранних (I-II) стадиях [3].

Показатели выживаемости пациентов уже на II стадии НМРЛ существенно снижены (41\%) по сравнению с I стадией (71\%) [4]. Столь значительные различия связаны с характеристиками развития опухоли: размером, метастазированием в регионарные лимфоузлы и внутренние органы [4]. Самостоятельное значение имеет степень дифференцировки ее клеток (Grade). Общеизвестно снижение выживаемости пациентов с высокой (Grade 1), средней (Grade 2) и низкой (Grade 3) степенью их дифференцировки при НМРЛ [5].

До настоящего времени нет информативных лабораторных показателей, позволяющих выявить НМРЛ на ранних стадиях. Чаще других с этой целью определяется концентрация фрагмента цитокератина 19 (CYFRA 21-1). Однако его диагностическая чувствительность при выявлении НМРЛ составляет лишь 59\% [6]. Кроме того, имеются данные об отсутствии его информативности при дифференцировании стадий НМРЛ и степени дифференцировки опухоли $[7,8]$. Другой показатель, концентрация SCC (антиген плоскоклеточной карциномы) в сыворотке крови, используется главным образом для ПКРЛ. Но и в этом случае его диагностическая чувствительность не превышает 41\% [9].

Между тем одной из центральных составляющих онкогенеза является опухолеассоциированное воспаление, оказывающее существенное влияние на рост и метастазирование опухоли [10]. Иммунные клетки микроокружения опухоли продуцируют хемокины, которые принимают участие в пролиферации клеток, ангиогенезе и метастазировании [11].

Интерлейкин-8 (CXCL8) принадлежит к семейству CXC-хемокинов, ответственных за привлечение иммунных клеток в зону воспаления. Взаимодействие CXCL8 с его рецептором CXCR1 усиливает опухолевый рост [12].

Высокая экспрессия CXCR1 в опухолевой ткани ассоциирована с плохим прогнозом у пациентов с НМРЛ [13]. Имеются данные о корреляции его уровня в клетках рака щитовидной железы с наличием метастазов в регионарные лимфоузлы [14], подавление сигнальных путей, запускаемых при активации CXCR1, приводит к торможению развития рака желудка [15].

Синтез провоспалительных хемокинов и их рецепторов клетками характерен не только для опухоли, но и для крови. Логично предположить, что рост их уровня в опухолевой ткани влияет на их концентрацию в крови. Однако в литературе отсутствуют данные об определении уровня CXCR1 в гранулоцитах, лимфоцитах и моноцитах крови в диагностике НМРЛ.

Сведения о взаимосвязи CXCL8 в сыворотке крови с характеристиками злокачественных новообразований, в том числе НМРЛ, немногочисленны и противоречивы. Отмечен повышенный уровень CXCL8 в крови пациентов с раком молочной и предстательной желез, положительная коррелятивная связь со стадиями заболевания и наличием метастазов в регионарные лимфоузлы $[13,16]$. По другим данным, он, напротив, подавляет злокачественный рост при НМРЛ [17]. 


\section{- ЦЕЛЬ ИССЛЕДОВАНИЯ}

Оценка уровня CXCL8 и CXCR1 в периферической крови и выяснение его взаимосвязи с дескрипторами опухоли при АК и ПКРЛ для последующего их использования в диагностике НМРЛ.

\section{- МАТЕРИАЛЫ И МЕТОДЫ}

Обследовано 109 пациентов с ПКРЛ и 94 пациента с АК при первом поступлении их в стационар РНПЦ онкологии и медицинской радиологии им. Н.Н. Александрова в период 2019-2021 гг. Характеристика пациентов представлена в табл. 1. В качестве группы контроля обследовано 40 человек без проявлений заболевания в возрасте 43-67 лет. Дополнительная группа сравнения включала 13 пациентов с доброкачественной опухолью легкого - гамартомой.

Кровь из локтевой вены пациентов и здоровых людей собирали натощак в вакутайнер с ЭДТА-К2 (Improvacuter, KHР). Для получения сыворотки кровь собирали в пробирку с тромбином и разделительным гелем (Improvacuter, KHP).

\section{Таблица 1}

Характеристика пациентов

Table 1

Patient's characteristics

\begin{tabular}{|c|c|c|}
\hline Характеристика / Characteristics & ПКРЛ / SCC & AK / AC \\
\hline \multicolumn{3}{|l|}{ Пол / Sex } \\
\hline Женский / F & 24 & 26 \\
\hline Мужской / М & 85 & 68 \\
\hline \multicolumn{3}{|l|}{ Стадия / Stage } \\
\hline 1 & 25 & 25 \\
\hline II & 43 & 29 \\
\hline III & 41 & 30 \\
\hline IV & 4 & 10 \\
\hline \multicolumn{3}{|l|}{ Размер опухоли / Tumor size } \\
\hline $\mathrm{T}<5 \mathrm{~cm}$ & 80 & 71 \\
\hline $\mathrm{T}>5 \mathrm{~cm}$ & 29 & 23 \\
\hline \multicolumn{3}{|c|}{ Метастазы в регионарные лимфоузлы / Lymph node metastases } \\
\hline No & 27 & 43 \\
\hline N1-3 & 82 & 51 \\
\hline \multicolumn{3}{|c|}{ Отдаленные метастазы / Distant metastases } \\
\hline Mo & 97 & 82 \\
\hline M1 & 12 & 12 \\
\hline \multicolumn{3}{|l|}{ Grade } \\
\hline G1 & 27 & 23 \\
\hline G2 & 51 & 39 \\
\hline G3 & 19 & 18 \\
\hline
\end{tabular}


Определение концентрации CYFRA 21-1 в сыворотке крови проводили методом иммунохемилюминесцентного анализа (ИХЛА) с помощью наборов реагентов на ИХЛА-анализаторе Cobas e411 (Rosche Diagnostics, США).

Определение концентрации CXCL8 и SCC в сыворотке крови пациентов и здоровых людей проводилось с помощью ИФА-наборов Fine Test (KHP) на автоматическом ИФА-анализаторе Brio (Seac, Италия) и планшетном фотометре Sirio (Seac, Италия) при длине волны 450 нм и референсной длине волны 620 нм.

Определение рецептора CXCR1 в клетках крови осуществляли на проточном цитофлуориметре Navios (Beckman Coulter, США). В пробирку помещали 100 мкл крови, стабилизированной ЭДТА-К2, и 10 мкл соответствующих антител, содержащих флуоресцентные метки: CD181(CXCR1)-PE/Cy5 (BioLegend, CША), CD14-FITC (Elabscience, CША), CD45-Pacific Orange (Exbio, Чехия). Через 15 мин. инкубации в темноте к смеси добавляли 1 мл лизирующего раствора VeraLyse (Beckman Coulter, Франция). Фиксацию антител на поверхности клеток проводили с помощью фиксирующего раствора IQTest 3 (Beckman Coulter, Франция).

Для всех выборок проверяли гипотезу нормальности распределения по критериям Колмогорова - Смирнова и Шапиро - Уилка. Поскольку значения показателей не подчинялись нормальному распределению, анализ проводили методами непараметрической статистики с использованием программных пакетов IBM SPSS Statistics 23 (IBM, CША) и MedCalc (США). Рассчитывались медиана и интерквартильный размах (25\% - 75\%). Для оценки различий между двумя независимыми группами применяли U-критерий Манна - Уитни. О взаимосвязи между определяемыми показателями и дескрипторами опухоли судили на основании расчета коэффициента ранговой корреляции Спирмена (R). Критическое значение уровня значимости принимали равным $5 \%$.

Оценку диагностической информативности биохимических тестов проводили с помощью характеристических кривых (ROC-анализ). B последующем вычисляли площадь под ROC-кривой (AUC, англ. Area Under Curve), на основании которой оценивали надежность определяемого показателя как потенциального биомаркера.

О диагностической ценности анализируемых показателей судили на основании расчета диагностической чувствительности (ДЧ), специфичности (ДС) и диагностической эффективности теста (ДЭ). Пороговое значение диагностического теста определяли как величину оптимального сочетания чувствительности и специфичности теста при построении ROC-кривых.

\section{- РЕЗУЛЬТАТЫ И ОБСУЖДЕНИЕ}

У пациентов с АК и ПКРЛ уровень CYFRA 21-1 был в 2 раза выше, чем в контрольной группе, уже на I стадии заболевания (табл. 2). Доля лимфоцитов C CXCR1 при аналогичном сравнении увеличилась примерно на 20\%. Уровень CXCL8 в сыворотке крови пациентов со II стадией АK и ПКРЛ возрастал более чем в два раза. Имелось значительное увеличение интенсивности флюоресценции комплексов антитело/рецептор (MFI), отражающей плотность расположения CXCR1 в мембранах лимфоцитов и гранулоцитов на I-II стадиях НMРЛ. При III-IV стадиях 
увеличение MFI было еще более выраженным. В то же время доля моноцитов, несущих рецептор CXCR1, существенно увеличивалась лишь на поздних стадиях заболевания. Сходным изменениям подвергалась и плотность расположения в них CXCR1, но лишь при ПКРЛ.

Имел место существенный подъем уровня SCC в сыворотке крови пациентов с ПКРЛ, но не АК. Уровень СYFRA 21-1 в сыворотке крови также увеличивался на ранних стадиях и в поздний период НМРЛ.

Практически отсутствовали существенные различия уровня всех определяемых показателей в крови пациентов с гамартомой легкого и здоровых людей. Напротив, у пациентов с ПКРЛ и АК он значительно отличался от такового при гамартоме (табл. 2).

Для показателей, продемонстрировавших существенное изменение уровня по сравнению со здоровыми людьми и пациентами с гамартомой на ранних стадиях АК и ПКРЛ и еще более - в поздний период, был рассчитан коэффициент корреляции Спирмена (r). Он подтвердил наличие связи со стадиями ПКРЛ и АК уровня CYFRA 21-1 (величина r для ПКРЛ и АК составила 0,469 и 0,329 соответственно), MFI CXCR1 (лимфоциты) ( $r=0,524$ и 0,526 соответственно) и доли лимфоцитов, снабженных CXCR1 ( $r=0,558$ и 0,662 соответственно).

У пациентов с размером опухоли более 5 см величина MFI CXCR1 (гранулоциты) была существенно выше по сравнению с опухолью меньшего размера ( $<5$ см) как у пациентов с АК, так и у пациентов с ПКРЛ (табл. 3). Этот же показатель значительно отличался у пациентов с метастазами в регионарные лимфоузлы (N1-3) по сравнению с N0.

Доля лимфоцитов с CXCR1 также повышалась в крови пациентов с АК и ПКРЛ (М1) по сравнению с М0. Ее величина также увеличивалась с ростом размера опухоли и снижением степени ее дифференцировки, но лишь у пациентов с ПКРЛ. Значения MFI CXCR1 (лимфоциты) возрастали у пациентов с размером опухоли >5 см и Grade 2, 3, но только при АК. Этот же показатель существенно повышался у пациентов с АК (M1) по сравнению с группой Мо.

По сути параллельно изменению концентрации CXCR1 содержащих клеткок крови в сыворотке крови пациентов обоих гистологических типов НМРЛ (М1) по сравнению с М0 вырос уровень лиганда для этого рецептора - CXCL8.

В ходе корреляционного анализа обнаруживается связь средней силы большинства упомянутых показателей с дескрипторами опухоли (табл. 3).

Концентрация CYFRA 21-1 при Т >5 см была выше у пациентов как с АК, так и с ПКРЛ. Только при ПКРЛ медиана значений этого показателя была статистически достоверно выше в группе пациентов М1 по сравнению с М0. Коэффициенты корреляции демонстрируют связь средней силы уровня CYFRA 21-1 с этими дескрипторами опухоли.

Полученные результаты свидетельствуют об однотипности изменений определяемых показателей в динамике АК и ПКРЛ. В частности, при обоих гистологических типах НМРЛ имеется схожее повышение медианы концентрации MFI CXCR1 (гранулоциты), доли лимфоцитов, снабженных рецептором CXCR1, в общей популяции этих клеток в крови, уровня CYFRA 21-1, тенденция к повышению уровня и всех остальных определяемых показателей по мере увеличения распространенности опухолевого процесса. 


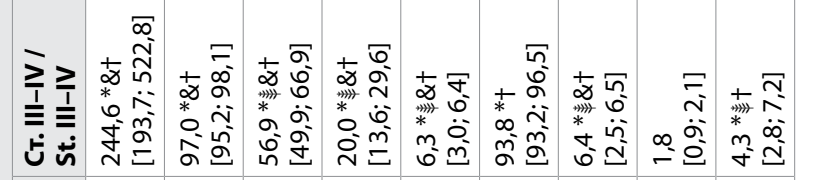

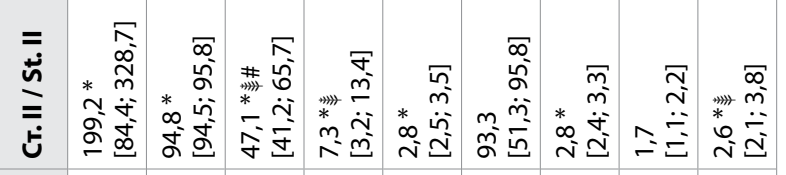

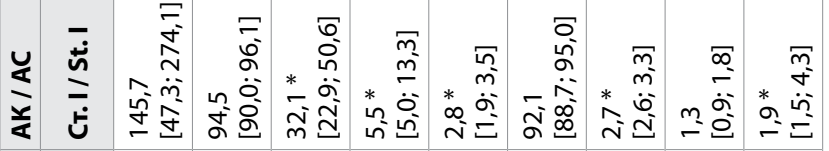

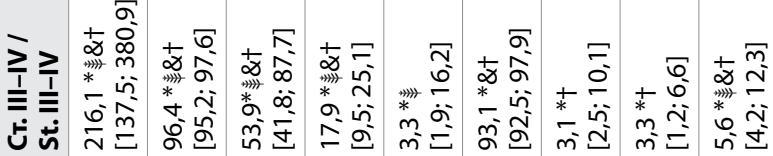

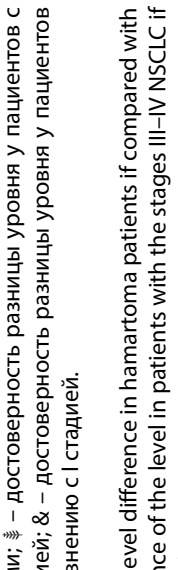

爱䒧

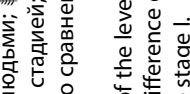

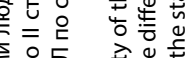

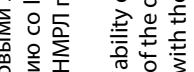

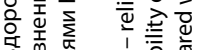

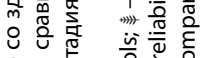

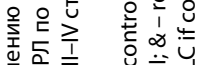

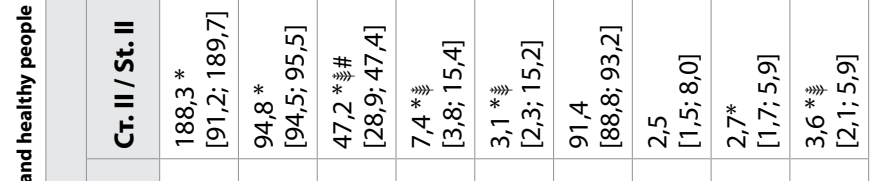

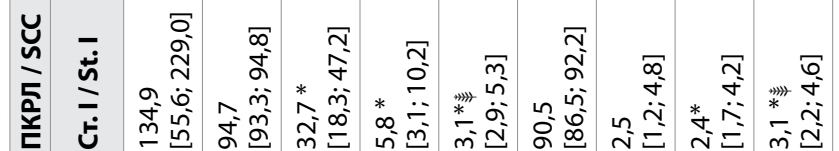

产

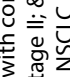

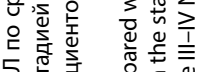

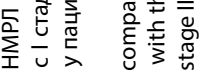

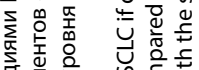

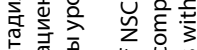

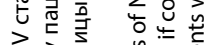

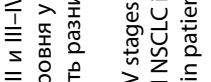

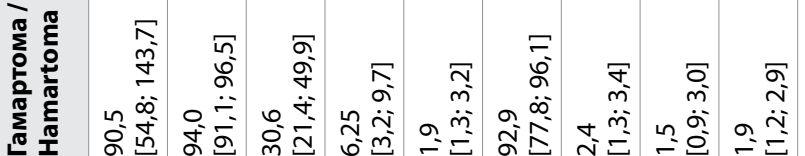

축 낭

桴 동

悉带过

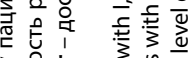

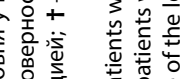

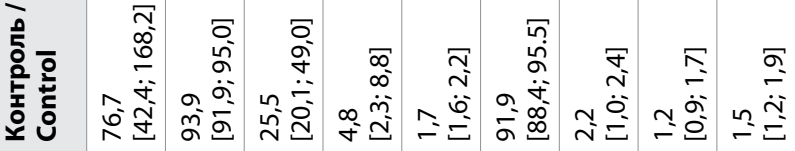

긍ํำ

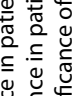

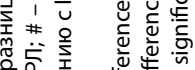

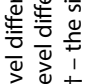

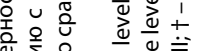

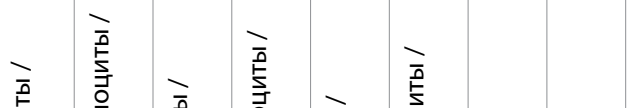

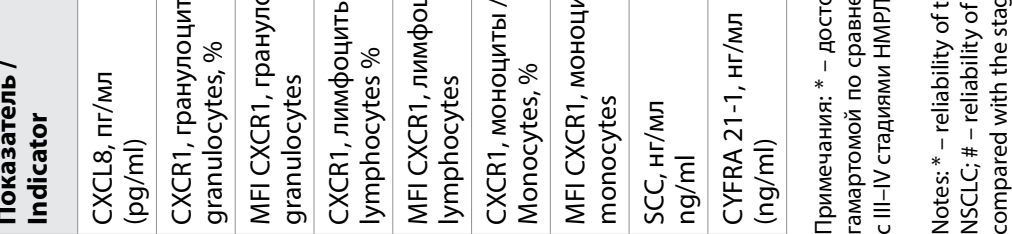




\section{Таблица 3}

Изменение концентрации CYFRA 21-1, CXCL8 и CXCR1 в крови пациентов в зависимости от характеристик НМРл

Table 3

Changes in the concentration of CYFRA 21-1, CXCL8, and CXCR1 in the blood of patients depending on the characteristics of NSCLC

\begin{tabular}{|c|c|c|c|c|c|c|}
\hline \multirow{2}{*}{ Показатель / Indicator } & \multicolumn{3}{|l|}{ ПКРЛ/SCC } & \multicolumn{3}{|l|}{ AK/AC } \\
\hline & $T<5 \mathrm{~cm}$ & $T>5 \mathrm{~cm}$ & $\mathbf{r}$ & $T<5 \mathrm{~cm}$ & $T>5 \mathrm{~cm}$ & $\mathbf{r}$ \\
\hline $\begin{array}{l}\text { MFI CXCR1, гранулоциты / } \\
\text { granulocytes }\end{array}$ & $\begin{array}{l}34,7^{*} \\
{[25,0 ; 52,6]}\end{array}$ & $\begin{array}{l}49,9 * \# \\
{[46,7 ; 72,9]}\end{array}$ & 0,378 & $\begin{array}{l}34,2 * \\
{[31,0 ; 60,2]}\end{array}$ & $\begin{array}{l}49,9^{*} \# \\
{[47,2 ; 67,8]}\end{array}$ & 0,373 \\
\hline $\begin{array}{l}\text { CXCR1, лимфоциты / } \\
\text { lymphocytes \% }\end{array}$ & $\begin{array}{l}12.2 * \\
{[3,8 ; 8,9]}\end{array}$ & $\begin{array}{l}20.4^{*} \# \\
{[9,1 ; 23,5]}\end{array}$ & 0,416 & - & - & - \\
\hline CYFRA 21-1, нг/мл (ng/ml) & $\begin{array}{l}3,7 * \\
{[2,2 ; 5.9]}\end{array}$ & $\begin{array}{l}5,4 * \# \\
{[4,2 ; 12,3]}\end{array}$ & 0,345 & $\begin{array}{l}2.8 * \\
{[1,6 ; 4,3]}\end{array}$ & $\begin{array}{l}5,8 * \# \\
{[2,5 ; 6,4]}\end{array}$ & 0,303 \\
\hline $\begin{array}{l}\text { MFI CXCR1, лимфоциты / } \\
\text { lymphocytes }\end{array}$ & - & - & - & $\begin{array}{l}2,8 * \\
{[2,3 ; 3,8]}\end{array}$ & $\begin{array}{l}5,0 * \# \\
{[3,1 ; 6,3]}\end{array}$ & 0,460 \\
\hline
\end{tabular}

Примечания: * - здесь и далее: статистическая значимость разницы уровня у пациентов с НМРЛ по сравнению со здоровыми людьми; \# - статистическая значимость разницы уровня у пациентов с размером опухоли $<5$ см и $>5$ см; $\mathrm{R}-$ коэффициент корреляции Спирмена.

Notes: * - here and after: statistical significance of the level difference in patients with NSCLC if compared with healthy people; \# - statistical significance of the level difference in patients with $\mathrm{T}<5 \mathrm{~cm}$ and $>5 \mathrm{~cm} ; \mathrm{r}$ - Spearman's correlation coefficient.

\begin{tabular}{|c|c|c|c|c|c|c|}
\hline \multirow{2}{*}{ Показатель / Indicator } & \multicolumn{3}{|l|}{ ПКРЛ } & \multicolumn{3}{|l|}{ AK } \\
\hline & NO & N1-3 & $\mathbf{r}$ & No & N1-3 & $\mathbf{r}$ \\
\hline $\begin{array}{l}\text { CXCR1 } \\
\text { granulocytes } \%\end{array}$ & $\begin{array}{l}94,3^{*} \\
{[94,5 ; 95,7]}\end{array}$ & $\begin{array}{l}95,4^{*} \# \\
{[95,2 ; 97,5]}\end{array}$ & 0,305 & - & - & - \\
\hline $\begin{array}{l}\text { MFI CXCR1, гранулоциты/ } \\
\text { granulocytes }\end{array}$ & $\begin{array}{l}34,2 * \\
{[25,1 ; 47,2] *}\end{array}$ & $\begin{array}{l}48,6^{*} \# \\
{[47,8 ; 74,9] * \#}\end{array}$ & 0,514 & $\begin{array}{l}33,9 * \\
{[27,5 ; 54,6]}\end{array}$ & $\begin{array}{l}49,4^{*} \# \\
{[47,2 ; 65,7]}\end{array}$ & 0,428 \\
\hline $\begin{array}{l}\text { CXCR1, лимфоциты / } \\
\text { lymphocytes \% }\end{array}$ & - & - & - & $\begin{array}{l}10,3^{*} \\
{[9,8 ; 20,4]}\end{array}$ & $\begin{array}{l}16,4^{*} \# \\
{[13,3 ; 28,8]}\end{array}$ & 0,328 \\
\hline
\end{tabular}

Примечание: \# - статистическая значимость разницы уровня у пациентов с наличием метастазов в регионарные лимфоузлы и пациентов без таковых.

Note: \# - statistical significance of the level difference in N1-3 patients if compared with N0.

\begin{tabular}{|c|c|c|c|c|c|c|}
\hline \multirow{2}{*}{ Показатель / Indicator } & \multicolumn{3}{|l|}{ ПКРЛ } & \multicolumn{3}{|l|}{ AK } \\
\hline & MO & M1 & $r$ & MO & M1 & $\mathbf{r}$ \\
\hline CXCL8, пг/мл (pg/ml) & $\begin{array}{l}187,5 * \\
{[98,3 ; 219,5]}\end{array}$ & $\begin{array}{l}400,7^{*} \# \\
{[140,2 ; 739,5]}\end{array}$ & 0,318 & $\begin{array}{l}198,7 * \\
{[195,7 ; 326,7]}\end{array}$ & $\begin{array}{l}305,8 * \# \\
{[229,7 ; 591,2]}\end{array}$ & 0,284 \\
\hline $\begin{array}{l}\text { CXCR1, лимфоциты / } \\
\text { lymphocytes, \% }\end{array}$ & $\begin{array}{l}12,8^{*} \\
{[4,8 ; 20,4]}\end{array}$ & $\begin{array}{l}20,9^{*} \# \\
{[22,3 ; 18,8]}\end{array}$ & 0,398 & $\begin{array}{l}13,3^{*} \\
{[7,3 ; 24,8]}\end{array}$ & $\begin{array}{l}21,8^{*} \# \\
{[15,6 ; 29,1]}\end{array}$ & 0,352 \\
\hline CYFRA 21-1, нг/мл (ng/ml) & $\begin{array}{l}2,8^{*} \\
{[1,6 ; 5,2]}\end{array}$ & $\begin{array}{l}5,9^{*} \# \\
{[1,7 ; 10,9]}\end{array}$ & 0,323 & - & - & - \\
\hline $\begin{array}{l}\text { MFI CXCR1, лимфоциты/ } \\
\text { lymphocytes }\end{array}$ & - & - & - & $\begin{array}{l}2,8^{*} \\
{[2,5 ; 3,7]}\end{array}$ & $\begin{array}{l}6,3^{*} \# \\
{[5,6 ; 6,4]}\end{array}$ & 0,511 \\
\hline
\end{tabular}

Примечание: \# - статистическая значимость разницы уровня у пациентов M1 по сравнению с Мо.

Note: \# - statistical significance of the level difference in M1 patients if compared with M0. 


\begin{tabular}{|c|c|c|c|c|c|c|c|c|}
\hline \multicolumn{9}{|c|}{ Окончание таблицы 3} \\
\hline \multirow{2}{*}{$\begin{array}{l}\text { Показатель / } \\
\text { Indicator }\end{array}$} & \multicolumn{4}{|l|}{ ПКРЛ/SCC } & \multicolumn{4}{|l|}{ AK/AC } \\
\hline & G1 & G2 & G3 & $\mathbf{r}$ & G1 & G2 & G3 & $\mathbf{r}$ \\
\hline $\begin{array}{l}\text { CXCR1, } \\
\text { лимфоциты / } \\
\text { lymphocytes, \% }\end{array}$ & - & - & - & - & $\begin{array}{l}11,1^{*} \\
{[6,8 ; 21,7]}\end{array}$ & $\begin{array}{l}14,3^{*} \# \\
{[8,5 ; 16,4]}\end{array}$ & $\begin{array}{l}28,4^{*} \# \S \\
{[17,9 ;} \\
29,6]\end{array}$ & 0,545 \\
\hline $\begin{array}{l}\text { MFI CXCR1, } \\
\text { лимфоциты / } \\
\text { lymphocyte }\end{array}$ & $\begin{array}{l}32,1^{*} \\
{[17,7 ; 51,6]}\end{array}$ & $\begin{array}{l}40,8^{* \#} \\
{[29,7 ; 54,2]}\end{array}$ & $\begin{array}{l}48,3^{*} \# \S \\
{[37,5 ; 65,1]}\end{array}$ & 0,363 & - & - & - & - \\
\hline
\end{tabular}

Примечания: \# - статистическая значимость разницы уровня у пациентов со средней и низкой степенью дифференцировки опухоли и высокой степенью дифференцировки опухоли; §-статистическая значимость разницы уровня у пациентов с низкой и средней степенью дифференцировки опухоли.

Notes: \# - statistical significance of the level difference in G2, 3 patients if compared with G1; § - statistical significance of the difference of the level in $\mathrm{G} 2$ and $\mathrm{G} 3$ patients.

Схожесть изменений уровня компонентов оси CXCL8/CXCR1 при AK и ПКРЛ указывает на возможность дальнейшей оценки их диагностической значимости в общей группе пациентов с НМРЛ.

Результаты ROC-анализа показывают, что значение MFI CXCR1 (гранулоциты) 10,9 позволяет отличить пациентов с I и II стадиями НМРЛ от здоровых людей с чувствительностью 98,1\%, специфичностью $71,1 \%$ и диагностической эффективностью 82,9\%. Площадь под ROC-кривой (AUC) MFI CXCR1 (гранулоциты) составляла 0,881, что соответствует хорошему качеству созданной модели. В то же время значение этого показателя >44,2 соответствует поздним стадиям НМРЛ с чувствительностью 87,5\% и специфичностью 93,3\% (табл. 4).

Превышение порогового значения (>2,8 нг/мл) CYFRA 21-1 позволяет верно выявить ранние стадии НМРЛ только в 68,4\% случаев с вероятностью истинно отрицательного результата 96,8\%. С другой стороны, для отличия поздних стадий от ранних расчетная величина пограничного значения этого показателя составила 4,1 нг/мл (чувствительность $79,2 \%$, специфичность - 68,4\%).

Доля лимфоцитов, снабженных CXCR1, при пороговом значении 2,9\% разделяет ранние стадии НМРЛ и здоровых людей с чувствительностью диагностической модели $80,0 \%$ и сравнительно невысокой специфичностью - 53,3\%.

Уровень CYFRA 21-1 не отличался в группах пациентов с I стадией НМРЛ по сравнению со II стадией, в то время как MFI CXCR1 (гранулоциты) при значениях этого показателя $>23,9$ соответствовал II стадии заболевания с чувствительностью $96,6 \%$, но низкой специфичностью $(57,1 \%)$.

Таким образом, в результате проведенного исследования установлено существенное увеличение концентрации CYFRA 21-1 в крови пациентов с НМРЛ по сравнению с доброкачественной опухолью легкого - гамартомой, а также со здоровыми людьми. Их уровень был выше контрольного уже на I стадии заболевания. В дальнейшем он возрастал и демонстрировал коррелятивную связь с размером опухоли при обоих гистологических типах НМРЛ (CYFRA 21-1), а при ПКРЛ еще и с наличием отдаленных метастазов. Эти данные, как и наблюдавшееся нами 
Диагностическая значимость определения уровня хемокина CXCL8

и его рецептора CXCR1 в крови пациентов с немелкоклеточным раком легкого

\section{Таблица 4}

Результаты ROC-анализа для существенно измененных показателей при НMРЛ, коррелятивно связанных с характеристиками опухоли

Table 4

The results of the ROC analysis for significantly changed parameters correlatively related to tumor characteristics

\begin{tabular}{|c|c|c|c|c|c|}
\hline \multirow{2}{*}{ Показатель / Indicator } & \multicolumn{5}{|c|}{ I-II ст. НМРЛ / здоровые (st. I-II NSCLC / controls) } \\
\hline & $\Pi 3 / \mathrm{CV}$ & ДЧ / TPR, \% & ДС / TNR, \% & AUC & ДЭ / АСС, \% \\
\hline $\begin{array}{l}\text { MFI CXCR1, гранулоциты / } \\
\text { granulocytes }\end{array}$ & 10,9 & 98,7 & 71,1 & 0,881 & 82,9 \\
\hline CXCR1 лимфоциты / lymphocytes, \% & 2,9 & 80,0 & 53,3 & 0,575 & 66,7 \\
\hline \multirow[t]{2}{*}{ CYFRA 21-1, нг/мл (ng/ml) } & 2,8 & 68,4 & 96,8 & 0,858 & 81,3 \\
\hline & \multicolumn{5}{|c|}{ I-II ст. НМРЛ / III-IV ст. НМРЛ (st. I-II NSCLC / st. III-IV NSCLC) } \\
\hline $\begin{array}{l}\text { MFI CXCR1, гранулоциты / } \\
\text { granulocytes }\end{array}$ & 44,2 & 87,5 & 93,3 & 0,876 & 90,0 \\
\hline \multirow[t]{2}{*}{ CYFRA 21-1, нг/мл (ng/ml) } & 4,1 & 79,2 & 68,4 & 0,768 & 80,6 \\
\hline & \multicolumn{5}{|c|}{ І ст. НМРЛ / ІІ ст. НМРЛ (st. I NSCLC / st. II NSCLC) } \\
\hline $\begin{array}{l}\text { MFI CXCR1, гранулоциты / } \\
\text { granulocytes }\end{array}$ & 23,9 & 96,6 & 57,1 & 0,732 & 76,7 \\
\hline
\end{tabular}

Примечания: ПЗ - пороговое значение; ДЧ - диагностическая чувствительность; ДС - диагностическая специфичность; дэ - диагностическая эффективность; AUC - площадь под ROC-кривой.

Notes: CV - cutoff value; TPR - diagnostic sensitivity;TNR - diagnostic specificity; ACC - accuracy of diagnostic test, \%; AUC - area under ROC-curve.

отсутствие разницы уровня этого биомаркера в группах пациентов с наличием и отсутствием метастазов в регионарные лимфоузлы, а также в зависимости от степени дифференцировки опухоли, согласуется с результатами, полученными другими исследователями [9].

Уровень CXCL8, хотя и претерпевал количественные изменения CXoжим образом, но при I стадии не отличался от контрольного. Это послужило основанием для того, чтобы не вовлекать его в оценку диагностической ценности его определения в дальнейшем.

Впервые были получены доказательства значительного увеличения относительного количества гранулоцитов и лимфоцитов, содержащих в своем составе CXCR1, а также плотности расположения рецептора во всех клетках лейкоцитарного ряда у пациентов с НМРЛ по сравнению со здоровыми людьми и пациентами с гамартомой. Он связывается с двумя хемокинами - CXCL8 и интерлейкином-6 [18]. Можно предположить, что увеличение презентации рецептора на клетках обусловлено ростом продукции его лигандов, что характерно для воспалительной реакции и развития раковой опухоли [12-16]. Наверное, поэтому в нашем исследовании MFI CXCR1 (гранулоциты) и относительное количество лимфоцитов, снабженных рецептором CXCR1, возрастали в зависимости от стадии заболевания.

Обнаруженные изменения дали основание оценить возможность использования рецептора CXCR1 в качестве маркера НМРЛ. С другой стороны, все определяемые биохимические параметры продемонстрировали сходные изменения в динамике АК и ПКРЛ, что позволило провести эту оценку в объединенной группе пациентов с НМРЛ, без учета гистологического типа. 
Результаты ROC-анализа продемонстрировали отсутствие информативности определения уровня CYFRA 21-1 для дифференциальной диагностики I и II стадий заболевания. Диагностическая эффективность определения MFI CXCR1 с этой целью составила 76,7\%. При этом чувствительность этого теста достаточно высока - 96,6\%, в то время как специфичность составила только 57,1\%. Вместе с тем получены доказательства сравнительно высокой диагностической ценности определения MFI CXCR1 (гранулоциты) у пациентов с I-II стадиями HМРЛ, пограничные значения которого существенно отличались от здоровых людей и пациентов с III-IV стадиями (чувствительность - 98,7\% и 87,5\%, специфичность - 71,1\% и 93,3\% соответственно). Это значительно превышает эффективность определения с этой целью концентрации CYFRA 21-1 в сыворотке крови.

Участие авторов: концепция и дизайн исследования, редактирование текста - А.Д. Таганович, сбор материала, обработка, написание текста - Д.И. Мурашко, Н.Н. Ковганко.

Конфликт интересов. Авторы заявляют об отсутствии конфликта интересов.

Conflict of interest. The authors declare no conflict of interest.

\section{ЛИТЕPATYPA/REFERENCES}

Brett C Bade (2020) Lung Cancer 2020: Epidemiology, Etiology and Prevention. Clin Chest Med, vol. 41, no 1, pp. 1-24. doi: 10.1016/j.ccm.2019.10.001.

2. Zappa C, Mousa SA (2016) Non-small cell lung cancer: current treatment and future advances. Trans/ Lung Cancer Res, vol. 5, no 3, pp. 288-300. doi: 10.21037/tlcr.2016.06.07 Brittany Ciupka (2020) Small Cell Lung Cancer vs. Non-small cell lung cancer: What's the Difference? National Foundation for Cancer Research (electronic journal), Nov 4, 2020. Avaliable at: https://www.nfcr.org/blog/small-cell-lung-cancer-vs-non-small-cell-lung-cancer-whats-the-difference/ (assessed 22.04.2021)

4. Shah R. (1996) Results of surgical treatment of stage I and II lung cancer. J Cardiovasc Surg (Torino), vol. 37, no 2, pp. 169-172.

5. Yasukawa M. (2018) Histological grade: analysis of prognosis of non-small cell lung cancer after complete resection. In Vivo, vol. 32, no 6, pp. 1505-1512. doi: 10.21873/ invivo.11407

6. Wieskopf B. (1995) CYFRA 21-1 as a biologic marker of non-small cell lung cancer. Evaluation of sensitivity, specificity and prognostic role. Chest, vol. 108, no 1, pp. 163-169. doi: 10.1378/chest.108.1.163.

7. Suzuki H. (2007) Preoperative CYFRA 21-1 levels as a prognostic factor in c-stage I non-small cell lung cancer. European Journal of Cardio-Thoracic Surgery, vol. 32 no 4, pp. 648-652.

8. Alkotyfan K. (2010) CYFRA 21-1 as a tumor marker for follow-up of patients with squamous cell carcinoma of oropharynx. Anticancer Res, vol. 30, no 6, pp. 2291-2296.

9. Lei Lui (2012) Clinical significance of CYFRA 21-1, SCC-Ag and telomerase activity in serum and pleural infusion of patients with squamous-cell lung cancer. Bioanalysis, vol. 4 no 19, pp. 2367-2374. doi: 10.4155/bio.12.203.

10. Gomes M. (2014) The role of inflammation in lung cancer. Adv Exp Med Biol, vol. 816, pp. 1-23. doi: 10.1007/978-3-0348-0387-8_1.

11. Sean Blandin Knight (2017) Progress and prospects of early detection of lung cancer. Open Biol, vol. 7, no 9, p. 170070. doi: 10.1098/rsob.170070.

2. Lui Q. (2016) The CXCR1/CXCR2 pathways in cancer. Cytokine Growth Factor Rev, vol. 31, pp. 61-71. doi: 10.1016.cytogfr.2016.08.002.

13. Yang F. (2021) CXCR1 correlates to poor outcomes of EGFR-TKI against advanced non-small cell lung cancer by activating chemokine and JAK-STAT pathway. Plum Pharmacol Ther., vol. 67, p. 102001. doi:10.1016/j.pupt.2021.102001.

14. Tang C. (2014) High expression of GPER1, EGFR and CXCR1 is associated with lymph node metastasis in papillary thyroid carcinoma. Int J Clin Exp Pathol, vol. 7, no 6 , pp. 3213-3223.

15. Wang J. (2016) Repertaxin, an inhibitor of the chemokine receptor CXCR1 and CXCR2, inhibits malignant behavior of human gastric cancer MKN45 cells in vitro and in vivo and enhances efficacy of 5-ftoruracil. Vol. 48, no 4, pp. 1341-1352. doi: 10.3892/ijo.2016.3371.

16. Yunfeng MA [et al] (2017) IL-6, IL-8 and TNF-a levels correlate with disease stage in breast cancer patients. Adv Clin Exp Med, vol. 26, no 3, pp. 421-426. doi: 10.17219/ acem/62120.

17. Wang J. (1996) Interleukin-8 inhibits non-small cell lung cancer proliferation: a possible role for regulation of tumor growth by autocrine and paracrine pathways. $J$ /nterferon Cytokine Res, vol. 16, no 1, pp. 53-60. doi: 10.1089/jir.1996.16.53.

18. Ha Helen (2017) Role of the CXCL8-CXCR1/2 Axis in Cancer and Inflammatory Diseases. Theranostics, vol. 7, no 6, pp. 1543-1588. doi: 10.7150/thno.15625.

Подана/Submitted: 28.04 .2021

Принята/Accepted: 11.05.2021

Контакты/Contacts: sherstyanoymurovei@mail.ru 